\title{
Progenitor Cells of the Mandibular Condylar Cartilage
}

\author{
Jennifer Robinson ${ }^{1,2} \cdot$ Alina O'Brien ${ }^{3} \cdot$ Jing Chen $^{1} \cdot$ Sunil Wadhwa $^{1}$
}

Published online: 10 July 2015

(C) Springer International Publishing AG 2015

\begin{abstract}
The secondary cartilage of the mandibular condyle is unique as it undergoes endochondral ossification during growth and robustly remodels in response to changes in its mechanical loading environment. This cartilage is derived from mesenchymal progenitor cells that express markers of early osteoblast differentiation, namely alkaline phosphatase (ALP) and runt-related transcription factor 2 (Runx2). Interestingly, these progenitor cells then differentiate into cartilage with appropriate mechanical loading. Our laboratory has determined that these cells can be labeled by osteoblast progenitor cell markers, including the 3.6 fragment of the rat collagen type 1 . However, the role these mesenchymal progenitor cells play in adult mandibular condylar cartilage maintenance and adaptation, as well as the existence of a more potent
\end{abstract}

This article is part of the Topical Collection on Molecular Biology of Adult Stem Cells

Sunil Wadhwa

Sw2680@cumc.columbia.edu

Jennifer Robinson

Jlr2228@columbia.edu

Alina O'Brien

Ano2108@cumc.columbia.edu

Jing Chen

Jc3835@cumc.columbia.edu

1 Division of Orthodontics, Columbia University College of Dental Medicine, 622 W. 168th Street, VC 9-219, New York, NY 10032, USA

2 Department of Biomedical Engineering, Columbia University, 351 Engineering Terrace, 1027 Amsterdam Avenue, New York, NY 10027, USA

3 Columbia University College of Dental Medicine, 630 W. 168th Street, New York, NY 10032, USA progenitor cell population within the mandibular condylar cartilage, remains in question. Further characterization of these cells is necessary to determine their potency and regenerative capacity to elucidate their potential for regenerative therapy.

Keywords TMJ $\cdot$ Mandibular condylar cartilage ·

Osteoprogenitor $\cdot$ Fibrocartilage

\section{Introduction}

The National Institute of Dental and Craniofacial Research (NIDCR) division of the National Institutes of Health (NIH) reported that temporomandibular joint (TMJ) disease is the second most common musculoskeletal disease in the USA. In the USA, $6-12 \%$ of the adult population suffers from TMJ disorder symptoms [1]; $10 \%$ of the patients with TMJ disorder suffer from degeneration of the joint and resulting pain, requiring joint replacement in severe cases [2]. To better design treatment and regenerative therapies, increased understanding of the progenitor cell population's function and role in TMJ remodeling is necessary. In this review, we will examine the unique properties of the mandibular condylar cartilage (MCC) and describe the identification of a mesenchymal progenitor cell population within the TMJ.

\section{Mandibular Condylar Cartilage Anatomy}

The MCC, located on the articular surface of the condyle, is composed of four distinct zones. The most superficial layer is the articular or superficial zone (Fig. 1a). It is found adjacent to the joint cavity and forms the outermost functional surface. Cells in this zone express the superficial zone protein (Szp), a product of the proteoglycan 4 gene [3]. The second zone is the 


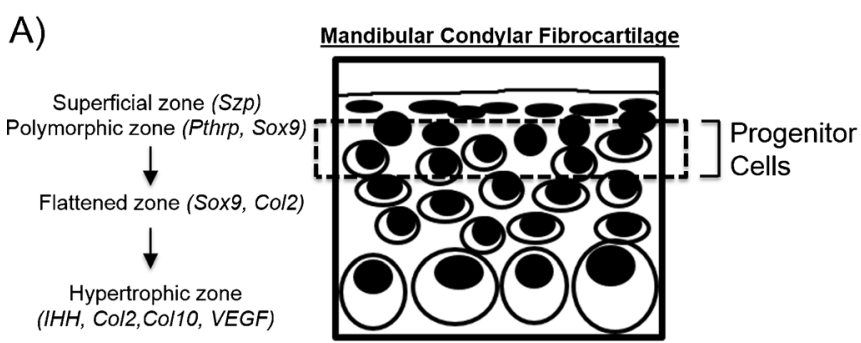

Fig. 1 a The mandibular condylar cartilage is comprised of four zones. Mesenchymal progenitor cells are found in the polymorphic zone and can be marked by the $3.6-\mathrm{kb}$ fragment of the rat collagen type I promoter (3.6

polymorphic or proliferative zone and is predominantly populated with chondroprogenitor cells. There is no structured organization in the formation or the arrangement of cells in this layer, in contrast to the organization and morphology within the growth plate cartilage [4] (see comment). Cells in this zone are actively proliferating and express parathyroidrelated protein (PTHrP) and SRY-box containing gene 9 (Sox9) [5]. PTHrP expression levels are mechanically regulated within the MCC [6]. PTHrP is believed to enhance or sustain proliferation of chondroprogenitor cells [5], promote the maturation of chondroprogenitor cells to chondroblasts in the flattened zone, and inhibit chondrocyte hypertrophy [6-8]. Sox9, on the other hand, is believed to be an important regulator of chondrogenesis [9]. In addition, PTHrP phosphorylates Sox 9 and enhances its ability to transactivate the collagen type II (Col2) promoter [7]. The third zone is the flattened zone. Cells in this layer are characterized by the expression of Sox 9 and Col2 [5]. The fourth zone, known as the hypertrophic zone, is characterized by cells that express and produce collagen type X (Col10) [5]. Unlike other growth plate cartilages, the MCC does not fuse and has an articulating surface.

After the cessation of growth, the mandibular condylar cartilage becomes phenotypically similar to other articular cartilages by entering into a post-mitotic state $[10,11]$. However, there still appears to be a progenitor cell population capable of reactivating in response to changes in mechanical loading [12]. Therefore, greater understanding and identification of the mandibular condylar cartilage progenitor cells is required in order to maintain and prevent the degeneration of the mature mandibular condylar cartilage over its lifetime and provide a target cell population for regeneration post injury or disease.

\section{Mandibular Condylar Cartilage Is Secondary Cartilage Derived From Periosteum}

The mandibular condylar cartilage is a secondary cartilage which has a distinct embryonic origin and development distinguishable from other primary cartilages [13]. Primary cartilage is composed solely of collagen type II and grows via

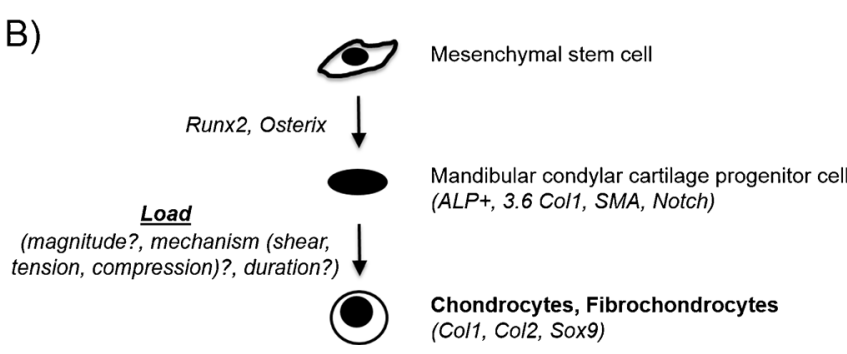

Col1) and/or the alpha-smooth muscle actin ( $\alpha$-SMA) promoter. b Mesenchymal progenitor cells require mechanical loading cues to differentiate into fibrocartilage

interstitial cell proliferation. In contrast, secondary cartilage is composed of both collagen types I and II and grows via appositional proliferation [14].

There is strong evidence that the mandibular condylar cartilage is derived from periosteum [15]. Supporting this theory, the mandibular condylar cartilage has been shown to derive from cells that express markers of early osteoblast differentiation, namely alkaline phosphatase (ALP) and runt-related transcription factor 2 (Runx2) [16•, 17]. The earliest sign of the MCC formation is observed at day 13 in mouse fetuses, when mesenchymal cell condensation forms the anlage of the future articular process [18]. The condylar cartilage develops from ALP-expressing progenitor cells which are continuous with the ALP-positive cells of the periosteum of the ossifying mandible [18-22]. Furthermore, the newly formed chondrocytes derived on day 15 of development all expressed ALP [21], which tapered on day 16 as the zones of the cartilage became distinct. This transitional pattern distinguishes the MCC, and possibly other secondary cartilages, from endochondral ossification in the long bones.

The role of Runx 2 in the development of the condylar cartilage is one of the most crucial pieces of data illustrating the phenotype and differentiation capacity of MCC progenitor cells. Runx 2 is a transcription factor necessary for bone formation [23-26] but does not inhibit the formation of primary cartilage $[23,25,26]$. Interestingly, Runx 2 is essential for the formation of the secondary cartilage of the mandibular condyle [16•]. Mice deficient in Runx2 failed to develop the MCC. These mice lacked collagen type II and aggrecan gene expression compared to the wild-type age-matched control (gestational day 18.5), which exhibited clearly formed condylar cartilage. It has been further shown that Sox9-expressing cells of the mandibular condylar cartilage are derived from Runx2/osterix-expressing cells within the mandibular condylar cartilage anlage [27]. In contrast, in growth plate cartilages, Runx2-expressing cells are derived from Sox9-expressing progenitor cells [28]. Therefore, the reversal expression pattern of Runx 2 and Sox9 is possibly a unique feature of mandibular condylar cartilage formation [27]. Together, these studies demonstrate the necessity for MCC progenitor cells to initially differentiate down an osteogenic lineage prior to 
chondrogenic differentiation for the development of functional condylar cartilage.

\section{Condylar Cartilage Progenitor Cells Can Differentiate Into Bone and Cartilage}

The cells of the prechondroblastic layer of the polymorphic zone are of great importance because they are the origin of nearly all cell divisions of the MCC cells [29-31]. Gene expression analysis shows that the progenitor cells are capable of differentiating into cells of an osteogenic or chondrogenic lineage [32•]. However, whether these cells are predifferentiated osteochondroprogenitors or true multipotent stem cells remains to be determined.

A thorough study of the specific expression of osteogenic lineage genes compared to chondrogenic lineage genes was conducted on a 2-day-old murine perichondrium and MCC [33॰]. The cartilage exhibited elevated levels of typical cartilage genes, including procollagen X and XI, matrix metalloproteinase (MMP) 9 and 13, aggrecan, and Indian hedgehog (IHH). On the other hand, the cells within the perichondrium expressed osteogenic lineage markers such as ALP and procollagen XIV [34] and cell-fate mediators such as TGF- $\beta$ and Notches 1, 3, and 4. Notch proteins are transmembrane receptors involved in cell-cell signaling which determines cell fate [35]. Within the perichondrium of MCC, Notch 1 has been implicated for its role in stimulating the proliferation and division of prechondroblasts [36]. Interestingly, the same receptor has been shown to play a role in suppressing the differentiation and proliferation of the perichondrium progenitor cells in the forelimb of murine embryos during development [37]. These opposing results further highlight the uniqueness and differing developmental pattern of the MCC compared to other cartilages formed via endochondral ossification. The authors also discovered increased expression of the tooth-associated genes (tuftelin, tuftelin-interacting protein 11, and dentin sialophosphoprotein), VEGF-B, and the muscle-specific transcription factor myogenic factors 5 and 6. The additional upregulation of these genes suggests an enhanced plasticity of the progenitor cells within the perichondrium. It is currently unclear whether this is a pure population of cells or a composition of multiple different cell phenotypes. Future studies focused on fluorescence-activated cell sorting (FACS) analysis can elucidate the population distribution based on the expression of each gene.

\section{Identification of Mandibular Condylar Cartilage Progenitor Cells}

Our group was one of the first to identify a MCC progenitor cell population. In designing our study, we knew that various fragments of the rat Colla1 mRNA promoter were initially constructed to mark cells of the osteoblast lineage. It was determined that the 3.6-kb fragment of the rat Colla1 (3.6 Col1) promoter marked earlier cells in the osteoblast lineage [38]. Due to the periosteal origin of the MCC, we sought to determine if the 3.6 Co1 fragment also marked a MCC progenitor cell population. We found that $3.6 \mathrm{Col} 1$ was predominantly located in the polymorphic zone of the MCC. To determine the fate of $3.6 \mathrm{Coll}$ cells in the $\mathrm{MCC}$, transgenic mice containing the Col 3.6-cre recombinase transgene were bred with Cre reporter mice (ROSA26). LacZ expression determined by X-gal staining was found in all of the zones of the MCC but not in the knee articular cartilage. However, not all of the cells in the flattened and hypertrophic zones of the MCC were X-gal stained. We also found that isolated 3.6 Coll cells had the potential to differentiate into fat and bone [32•], suggesting that mandibular condylar cartilage progenitor cells exhibit similar potency compared to mesenchymal stem cells. A recent report has shown a mesenchymal origin for mandibular condylar cartilage progenitor cells further supporting this hypothesis.

Recently, a report has further illustrated the mesenchymal origin of mandibular condylar cartilage progenitor cells. Cellfate mapping experiments revealed that cells expressing alpha-smooth muscle actin ( $\alpha$-SMA), another marker of osteoblast precursors [39], were the precursors for future mandibular condylar cartilage hypertrophic cells [40]. Successful differentiation of these cells into other mesenchymal tissues (e.g., muscle), and not to tissues of other germ layer lineages, is needed in order to confirm mesenchymal origin of these progenitor cells.

\section{Progenitor Cells Require Mechanical Loading for Differentiation Into Cartilage}

It now appears that MCC progenitor cells are mesenchymal progenitor cells, which have been diverted to chondrogenesis in response to mechanical loading (Fig. 1b) [33•]. Early evidence in chick embryo development studies illustrated the development of secondary cartilage in membrane bones only when the articulations were mobile and experienced mechanical strain. In subsequent studies with paralyzed embryos, cartilage did not form, and the progenitor cells produced bone instead [41]. Similar results have been recently shown in mice [42]. Future studies must address the specific parameters of mechanical force (i.e., duration, magnitude, direction) required to promote differentiation into physiologically relevant mandibular condylar cartilage cells. This information is vital in maintaining healthy condylar cartilage and stimulating new growth post injury or disease. 


\section{Summary and Conclusions}

Collectively, recent evidence details the population of mesenchymal progenitor cells located at the superior surface of the mandibular condylar cartilage that can be labeled by $3.6 \mathrm{Col} 1$ and/or $\alpha$-SMA. These cells then undergo endochondral ossification in young growing mice in response to mechanical loading. This increased knowledge of MCC progenitor cell phenotype is advantageous for determining cell marker targets for in vitro culturing and implanting cells for MCC regeneration. Cell biologists and tissue engineers must focus on attaining the correct cell phenotypes and priming the differentiation and regeneration capacity in tissue engineered constructs in vitro and in vivo. The recent understanding of progenitor cell phenotypes suggests that these cells could be primed for differentiation in vitro using Runx2 (and potentially Sox9) and mechanical loading to adequately generate anatomically correct MCC. However, many questions still remain. What is the nature of mechanical loading which activates chondrogenesis? What role does the mandibular condylar cartilage progenitor cells play in adult mice? What is the role of these progenitor cells during degeneration? Is there a more multipotent progenitor cell population that exists within the mandibular condylar cartilage? Answering these questions will unlock the regenerative capacity of these cells and provide understanding on treatment modalities to maintain healthy cartilage and stimulate new tissue formation post injury or disease.

Acknowledgments This work was supported by NIH NIDCR 2R56DE020097-06 (SW) and NIH NIDCR K12 (SW) TMJ Training Fellowship.

\section{Compliance with Ethics Guidelines}

Conflict of Interest Jennifer Robinson, Alina O'Brien, Jing Chen, and Sunil Wadhwa declare that they have no conflict of interest.

Human and Animal Rights and Informed Consent This article does not contain any studies with human or animal subjects performed by any of the authors.

\section{References}

Papers of particular interest, published recently, have been highlighted as:

\section{- Of importance}

1. Lipton JA, Ship JA, Larach-Robinson D. Estimated prevalence and distribution of reported orofacial pain in the United States. J Am Dent Assoc. 1993;124(10):115-21.

2. Tanaka E, Detamore MS, Mercuri LG. Degenerative disorders of the temporomandibular joint: etiology, diagnosis, and treatment. J Dent Res. 2008;87(4):296-307.
3. Ohno S et al. Expression of superficial zone protein in mandibular condyle cartilage. Osteoarthrit Cart. 2006;14(8):807-13.

4. Wadhwa S, Kapila S. TMJ disorders: future innovations in diagnostics and therapeutics. J Dent Educ. 2008;72(8):930-47.

5. Shibukawa $\mathrm{Y}$ et al. Temporomandibular joint formation and condyle growth require Indian hedgehog signaling. Dev Dyn. 2007;236(2):426-34.

6. Rabie $\mathrm{AB}$ et al. PTHrP regulates chondrocyte maturation in condylar cartilage. J Dent Res. 2003;82(8):627-31.

7. Huang $\mathrm{W}$ et al. The chondrogenic transcription factor Sox 9 is a target of signaling by the parathyroid hormone-related peptide in the growth plate of endochondral bones. Proc Natl Acad Sci U S A. 2001;98(1):160-5.

8. Tsutsui TW et al. Development of craniofacial structures in transgenic mice with constitutively active $\mathrm{PTH} / \mathrm{PTHrP}$ receptor. Bone. 2008;42(2):321-31.

9. Bi W et al. Sox9 is required for cartilage formation. Nat Genet. 1999;22(1):85-9.

10. Livne E, Weiss A, Silbermann M. Changes in growth patterns in mouse condylar cartilage associated with skeletal maturation and senescence. Growth Dev Aging. 1990;54(4):183-93.

11. Luder HU. Age changes in the articular tissue of human mandibular condyles from adolescence to old age: a semiquantitative light microscopic study. Anat Rec. 1998;251(4):439-47.

12. Bock NC, Ruf S. Class II division 2 treatment - does skeletal maturity influence success and stability? J Orofac Orthop. 2013;74(3): 187-204.

13. Delatte $\mathrm{M}$ et al. Primary and secondary cartilages of the neonatal rat: the femoral head and the mandibular condyle. Eur J Oral Sci. 2004;112(2):156-62.

14. Copray JC, Duterloo HS. A comparative study on the growth of craniofacial cartilages in vitro. Eur J Orthod. 1986;8(3):157-66.

15. Meikle MC. In vivo transplantation of the mandibular joint of the rat; an autoradiographic investigation into cellular changes at the condyle. Arch Oral Biol. 1973;18(8):1011-20.

16. Shibata $\mathrm{S}$ et al. Runx2-deficient mice lack mandibular condylar cartilage and have deformed Meckel's cartilage. Anat Embryol (Berl). 2004;208(4):273-80. This paper illustrates the vital role of Runx 2 in mandibular condylar cartilage development. Mice lacking Runx2 do not develop mandibular condylar cartilage.

17. Shibata $\mathrm{S}$ et al. Immunohistochemistry of collagen types II and X, and enzyme-histochemistry of alkaline phosphatase in the developing condylar cartilage of the fetal mouse mandible. J Anat. 1997;191(Pt 4):561-70.

18. Shibata $\mathrm{S}$ et al. A histological study of the developing condylar cartilage of the fetal mouse mandible using coronal sections. Arch Oral Biol. 1996;41(1):47-54.

19. Silbermann $M$ et al. Further characterisation of the extracellular matrix in the mandibular condyle in neonatal mice. J Anat. 1987;151:169-88.

20. Shibata $\mathrm{S}$ et al. In situ hybridization and immunohistochemistry of bone sialoprotein and secreted phosphoprotein 1 (osteopontin) in the developing mouse mandibular condylar cartilage compared with limb bud cartilage. J Anat. 2002;200(3):309-20.

21. Miyake T, Cameron AM, Hall BK. Stage-specific expression patterns of alkaline phosphatase during development of the first arch skeleton in inbred C57BL/6 mouse embryos. J Anat. 1997;190(2): 239-60.

22. Fukada $\mathrm{K}$ et al. In situ hybridisation study of type I, II, X collagens and aggrecan mRNAs in the developing condylar cartilage of fetal mouse mandible. J Anat. 1999;195(3):321-9.

23. Komori $\mathrm{T}$ et al. Targeted disruption of $\mathrm{Cbfa} 1$ results in a complete lack of bone formation owing to maturational arrest of osteoblasts. Cell. 1997;89(5):755-64. 
24. Otto $\mathrm{F}$ et al. Cbfa1, a candidate gene for cleidocranial dysplasia syndrome, is essential for osteoblast differentiation and bone development. Cell. 1997;89(5):765-71.

25. Inada $\mathrm{M}$ et al. Maturational disturbance of chondrocytes in Cbfa1deficient mice. Dev Dyn. 1999;214(4):279-90.

26. Hoshi K, Komori T, Ozawa H. Morphological characterization of skeletal cells in Cbfa1-deficient mice. Bone. 1999;25(6):639-51.

27. Shibata S, Yokohama-Tamaki T. An in situ hybridization study of Runx2, Osterix, and Sox9 in the anlagen of mouse mandibular condylar cartilage in the early stages of embryogenesis. J Anat. 2008;213(3):274-83.

28. Akiyama $\mathrm{H}$ et al. Osteo-chondroprogenitor cells are derived from Sox9 expressing precursors. Proc Natl Acad Sci U S A. 2005;102(41):14665-70.

29. Blackwood HJJ. Growth of the mandibular condyle of the rat studied with tritiated thymidine. Arch Oral Biol. 1966;11(5):493-500.

30. Lars F. Cellular kinetics within the mandibular joint. Acta Odontol Scand. 1967;25(5):437.

31. Luder HU, Leblond CP, von der Mark K. Cellular stages in cartilage formation as revealed by morphometry, radioautography and type II collagen immunostaining of the mandibular condyle from weanling rats. Am J Anat. 1988;182(3):197-214.

32. Chen $\mathrm{J}$ et al. Isolation and characterization of murine mandibular condylar cartilage cell populations. Cells Tissues Organs. 195(3): p. 232-43. This is one of the first papers that details the isolation and characterization of the progenitor cells of the mandibular condylar cartilage. The 3.6-kb fragment of the rat collagen type I promoter was utilized to isolate cells.

33. Hinton RJ, Serrano M, So S. Differential gene expression in the perichondrium and cartilage of the neonatal mouse temporomandibular joint. Orthod Craniofac Res. 2009;12(3):168-77. This paper discusses the potency theories for mandibular condylar cartilage. The gene expression studies of mandibular condylar cartilage progenitor cells detailed in the paper resulted in genes involved in differentiation of multiple cell lineages (bone, cartilage, teeth) and cell-fate (Notch).

34. Walchli $\mathrm{C}$ et al. Tissue-specific expression of the fibril-associated collagens XII and XIV. J Cell Sci. 1994;107:669-81.

35. Artavanis-Tsakonas S, Rand MD, Lake RJ. Notch signaling: cell fate control and signal integration in development. Science. 1999;284(5415):770-6

36. So S, Serrano M, Hinton R. Notch signaling in mandibular condylar cartilage. J Dent Res, 2007. Special Issue A: p. Abstract 3010.

37. Watanabe $\mathrm{N}$ et al. Suppression of differentiation and proliferation of early chondrogenic cells by Notch. J Bone Miner Metab. 2003;21(6):344-52.

38. Kalajzic I et al. Expression profile of osteoblast lineage at defined stages of differentiation. J Biol Chem. 2005;280(26):24618-26.

39. Kalajzic $Z$ et al. Use of an alpha-smooth muscle actin GFP reporter to identify an osteoprogenitor population. Bone. 2008;43(3): 501-10.

40. Yadav $\mathrm{S}$ et al. In vivo fate mapping identifies progenitor of mandibular condylar cartilage. in International Association for Dental Research. 2013. Seattle, Washington.

41. Murray P, Smiles M. Factors in the evocation of adventitious (secondary) cartilage in the chick embryo. Austral J Zool. 1965;13(3):351-82.

42. Jahan $\mathrm{E}$ et al. Fetal jaw movement affects Ihh signaling in mandibular condylar cartilage development: the possible role of Ihh as mechanotransduction mediator. Arch Oral Biol. 2014;59(10): $1108-18$. 